\title{
Isolation and Identification of Phenolic Compounds from the Root Bark of Morus alba L.
}

\author{
Jae-Woo Jung · Ji-Hae Park · Kyeong-Hwa Seo $\cdot$ Yoon-Su Baek · Eun-Ji Oh • \\ Dae-Young Lee · Dong-Wook Lim · Daeseok Han · Nam-In Baek*
}

\section{상백피(Morus alba root barks)로부터 페놀화합물의 분리 및 동정}

정재우·박지해 · 서경화 · 오은지 · 백윤수 - 이대영 · 임동욱·한대석 · 백남인*

Received: 4 February 2015 / Accepted: 17 March 2015 / Published Online: 30 June 2015

(C) The Korean Society for Applied Biological Chemistry 2015

\begin{abstract}
The root barks of Morus alba L. were extracted with $80 \%$ aqueous $\mathrm{MeOH}$, and the concentrated extract was partitioned with EtOAc, $n$ - $\mathrm{BuOH}$, and $\mathrm{H}_{2} \mathrm{O}$ fractions. The repeated silica gel, octadecyl silica gel, and Sephadex LH-20 column chromatographies of the EtOAc and $n$-BuOH fractions led to isolation of four phenolic compounds. The chemical structures of the compounds were determined as norartocarpanone (1), 2',4',7-trihydroxy-(2S)flavanone (2), methyl $\beta$-resorcylate (3), and ( $Z$ )-oxyresveratrol-4$O-\beta$-D-glucopyranoside (4). Compound 4 was isolated for the first time from the root barks of M. alba L.
\end{abstract}

Keywords Morus alba $\cdot$ nuclear magnetic resonance $\cdot$ phenolic compound $\cdot$ root bark $\cdot(Z)$-oxyresveratrol-4- $O-\beta$-D-glucopyranoside

J.-W. Jung $\cdot$ J.-H. Park $\cdot$ K.-H. Seo $\cdot$ E.-J. Oh $\cdot$ Y.-S. Baek $\cdot$ N.-I. Baek Graduate School of Biotechnology and Department of Oriental Medicine Biotechnology, Kyung Hee University, Yongin 446-701, Republic of Korea

D.-Y. Lee

Department of Herbal Crop Research, National Institute of Horticultural and Herbal Science, RDA, Eumseong 369-873, Republic of Korea

\section{D.W. Lim · D. Han}

Division of Matabolism and Functionality Research, Korea Food Research Institute, Sungnam 463-746, Republic of Korea

*Corresponding author (N.-I. Baek: nibaek@khu.ac.kr)

This is an Open Access article distributed under the terms of the Creative Commons Attribution Non-Commercial License (http://creativecommons. org/licenses/by-nc/3.0/) which permits unrestricted non-commercial use, distribution, and reproduction in any medium, provided the original work is properly cited.
상백피로부터 이차대사산물을 분리하고 각 물질에 대한 성분분 석을 통해 상백피의 의약품 및 기능성 화장품 소재로서의 활용 방안을 모색하는 실험을 수행하였다. 저자 등은 상백피로부터 용매추출, 용매분획, 및 column chromatography를 반복 실시하 여 5종의 triterpenoid 화합물을 분리 동정하여 보고한 바 있으 며(Jung 등, 2014), 이번 실험에서는 4종의 phenolic 화합물을 분리 동정하였다.

이전에 보고된 바와 같이 상백피에서 얻어진 EtOAc 분획 (122 g)으로부터 $\mathrm{SiO}_{2}$ column chromatography (c.c.) (12.5× $17 \mathrm{~cm}, \quad n$-hexane-EtOAc $=4: 1 \rightarrow 2: 1 \rightarrow 1: 1 \rightarrow$ chloroform $\left(\mathrm{CHCl}_{3}\right)$ $\mathrm{MeOH}=10: 1$, 각 $27 \mathrm{~L}$ )를 실시하여 41개의 분획물(MRE-1-MRE41)을 얻었다. MRE-28 [2.2 g, elution volume/total volume $(\mathrm{Ve} / \mathrm{Vt})=0.595-0.670]$ 을 ODS c.c. $\left(\varphi 7 \times 4 \mathrm{~cm}, \mathrm{MeOH}-\mathrm{H}_{2} \mathrm{O}=2: 1 \rightarrow\right.$ $4: 1 \rightarrow 6: 1$, 각 $2 \mathrm{~L}$ )를 실시하여 16 개의 분획(MRE-28-1-MRE-2816)으로 나누었다. MRE-28-2 (44 mg, $\mathrm{Ve} / \mathrm{Vt}=0.021-0.032)$ 를 Sephadex LH-20 c.c. $\left(\varphi 1 \times 60 \mathrm{~cm}, \mathrm{MeOH}-\mathrm{H}_{2} \mathrm{O}=4: 1,0.8 \mathrm{~L}\right)$ 를 실시하여 8 개의 분획(MRE-28-2-1-MRE-28-2-8)으로 나누었고, 그 중 화합물 1 (MRE-28-2-7, $26 \mathrm{mg}, \mathrm{Ve} / \mathrm{Vt}=0.478-0.578, \mathrm{TLC}$ $\left.\left(\mathrm{ODS} \mathrm{F}_{254 \mathrm{~S}}\right) \mathrm{R}_{\mathrm{f}} 0.61, \mathrm{MeOH}-\mathrm{H}_{2} \mathrm{O}=3: 1\right)$ 을 분리하였다. MRE-30 $(1.2 \mathrm{~g}, \mathrm{Ve} / \mathrm{Vt}=0.672-0.683)$ 을 ODS c.c. $\left(\varphi 2 \times 10 \mathrm{~cm}, \mathrm{MeOH}-\mathrm{H}_{2} \mathrm{O}\right.$ $=3: 2 \rightarrow 3: 1 \rightarrow 5: 1$, 각 $1.2 \mathrm{~L}$ )를 실시하여 24개의 분획(MRE-30-1MRE-30-24)으로 나누었고, 그 중 화합물 2 (MRE-30-3, $8 \mathrm{mg}$, $\mathrm{Ve} / \mathrm{Vt}=0.012-0.022$, TLC (ODS $\mathrm{F}_{254 \mathrm{~S}}$ ) $\mathrm{R}_{\mathrm{f}} 0.69, \mathrm{MeOH}-\mathrm{H}_{2} \mathrm{O}=2: 1$ )를 분리하였다. $\mathrm{MRB}(78 \mathrm{~g})$ 를 $\mathrm{SiO}_{2}$ c.c. $\left(\varphi 12 \times 20 \mathrm{~cm}, \mathrm{CHCl}_{3}-\right.$ $\mathrm{MeOH}-\mathrm{H}_{2} \mathrm{O}=9: 3: 1 \rightarrow 65: 35: 10 \rightarrow 6: 4: 1$, 각 $1.5 \mathrm{~L}$ )를 실시하여 15 개의 분획물(MRB-1-MRB-15)을 얻었다. MRB-1 $(790 \mathrm{mg}, \mathrm{Ve} / \mathrm{Vt}$ $=0.000-0.039)$ 을 $\mathrm{ODS}$ c.c. $\left(\varphi 3 \times 7 \mathrm{~cm}, \mathrm{MeOH}-\mathrm{H}_{2} \mathrm{O}=1: 1\right.$, 각 $\left.0.7 \mathrm{~L}\right)$ 를 실시하여 4개의 분획(MRB-1-1-MRB-1-4)으로 나누었고, 그 중 화합물 3 (MRB-1-2, $19 \mathrm{mg}, \mathrm{Ve} / \mathrm{Vt}=0.513-0.812$, TLC (ODS $\left.\mathrm{F}_{254 \mathrm{~S}}\right) \mathrm{R}_{\mathrm{f}}$ 0.57, MeOH-H $\left.\mathrm{H}_{2} \mathrm{O}=3: 2\right)$ 을 분리하였다. MRB-10 (2.3 g, $\mathrm{Ve} / \mathrm{Vt}=0.589-0.664)$ 을 ODS c.c. $\left(\varphi 4 \times 8 \mathrm{~cm}, \mathrm{MeOH}-\mathrm{H}_{2} \mathrm{O}=2: 3\right.$, 
<smiles>O=C1C[C@H](c2ccc(O)cc2O)Oc2cc(O)cc(O)c21</smiles>

norartocarpanone (1)<smiles>O=C1C[C@H](c2ccc(O)cc2O)Oc2cc(O)ccc21</smiles>

2',4',7'-trihydroxy-(2S)-flavanone (2)<smiles>COC(=O)c1ccc(O)cc1O</smiles><smiles>OCC1C(O)OC(Oc2ccc3c(O)c(O)cc(O)c3c2)C(O)(O)C1O</smiles>

methyl $\beta$-resorcylate (3) (Z)-oxyresveratrol-4-O- $\beta$-D-glucopyranoside (4)

Fig. 1 Chemical structures of phenolic compounds (1-4) from the root barks of Morus alba L.

각 $1.0 \mathrm{~L}$ )를 실시하여 8 개의 분획(MRB-10-1-MRB-10-8)으로 나 누었다. MRB-10-2 $(186 \mathrm{mg}, \mathrm{Ve} / \mathrm{Vt}=0.112-0.173)$ 를 $\mathrm{SiO}_{2}$ c.c. $\left(\varphi 3 \times 10 \mathrm{~cm}, \mathrm{CHCl}_{3}-\mathrm{MeOH}-\mathrm{H}_{2} \mathrm{O}=7: 3: 1\right.$, 각 $0.75 \mathrm{~L}$ )를 실시하여 4개의 분획(MRB-10-2-1-MRB-10-2-4)으로 나누었다. MRB-10$2-2(120 \mathrm{mg}, \mathrm{Ve} / \mathrm{Vt}=0.481-0.803)$ 를 Sephadex LH-20 c.c. $(\varphi$ $2 \times 60 \mathrm{~cm}, \mathrm{MeOH}-\mathrm{H}_{2} \mathrm{O}=4: 1$, 각 $\left.0.1 \mathrm{~L}\right)$ 를 실시하여 4 개의 분획 (MRB-10-2-1-1-MRB-10-2-2-4)으로 나누었고, 그 중 화합물 4 (MRB-10-2-2-4, $5 \mathrm{mg}$, Ve/Vt=0.720-1.000, TLC (ODS F $254 \mathrm{~s}$ ) $\left.\mathrm{R}_{\mathrm{f}} 0.43, \mathrm{MeOH}-\mathrm{H}_{2} \mathrm{O}=1: 1\right)$ 를 분리하였다.

화합물 1 (norartocarpanone): Yellow amorphous powder (in $\mathrm{MeOH}) ;[\alpha]_{\mathrm{D}}^{25}+12.4^{\circ}($ c $0.12, \mathrm{MeOH}) ;$ m.p. 248-253; EI/MS $\mathrm{m} / \mathrm{z} 288[\mathrm{M}]^{+}$; IR $\left(\mathrm{CaF}_{2}\right.$ plate, v) 3454, 1661, 1608, 1598 $\mathrm{cm}^{-1} ;{ }^{1} \mathrm{H}-\mathrm{NMR}\left(400 \mathrm{MHz}, \mathrm{CD}_{3} \mathrm{OD}, \delta_{\mathrm{H}}\right) 7.22(1 \mathrm{H}, \mathrm{d}, J=8.8$ $\left.\mathrm{Hz}, \mathrm{H}-6^{\prime}\right), 6.33\left(1 \mathrm{H}, \mathrm{dd}, J=8.8,2.4 \mathrm{~Hz}, \mathrm{H}-5^{\prime}\right), 6.32(1 \mathrm{H}, \mathrm{d}$, $\left.J=2.4 \mathrm{~Hz}, \mathrm{H}-3^{\prime}\right), 5.88(1 \mathrm{H}, \mathrm{d}, J=2.4 \mathrm{~Hz}, \mathrm{H}-6), 5.85(1 \mathrm{H}, \mathrm{d}$, $J=2.4 \mathrm{~Hz}, \mathrm{H}-8), 5.58(1 \mathrm{H}, \mathrm{dd}, J=13.2,2.8 \mathrm{~Hz}, \mathrm{H}-2), 3.03$ $(1 \mathrm{H}, \mathrm{dd}, J=17.2,13.2 \mathrm{~Hz}, \mathrm{H}-3 \mathrm{a}), 2.68(1 \mathrm{H}, \mathrm{dd}, J=17.2,2.8$ $\mathrm{Hz}, \mathrm{H}-3 \mathrm{~b}) ;{ }^{13} \mathrm{C}-\mathrm{NMR}\left(100 \mathrm{MHz}, \mathrm{CD}_{3} \mathrm{OD}, \delta_{\mathrm{C}}\right) 198.22$ (C-4), 169.18 (C-8a), 166.41 (C-7), 165.47 (C-5), 159.69 (C-4'), 156.76 (C-2'), 128.83 (C-6'), 117.93 (C-1'), 107.76 (C-3'), 103.40 (C-5'), 103.08 (C-4a), 97.18 (C-6), $96.41 \quad$ (C-8), 75.89 (C-2), 43.09 (C-3).

화합물 2 (2',4',7-trihydroxy-(2S)-flavanone): Red amorphous powder (in $\mathrm{MeOH}$ ); $[\alpha]_{\mathrm{D}}^{25}-10.2^{\circ}$ (c 0.39 , MeOH); m.p. 140-145; EI/MS $m / z 272[\mathrm{M}]^{+}$; IR $\left(\mathrm{CaF}_{2}\right.$ plate, v) 3460, $1668, \quad 1602,1591 \mathrm{~cm}^{-1} ;{ }^{1} \mathrm{H}-\mathrm{NMR} \quad\left(400 \mathrm{MHz}, \mathrm{CD}_{3} \mathrm{OD}, \delta_{\mathrm{H}}\right)$ $7.72(1 \mathrm{H}, \mathrm{d}, J=8.8 \mathrm{~Hz}, \mathrm{H}-5), 7.24\left(1 \mathrm{H}, \mathrm{d}, J=8.8 \mathrm{~Hz}, \mathrm{H}-6^{\prime}\right)$, $6.48(1 \mathrm{H}, \mathrm{dd}, J=8.8,2.4 \mathrm{~Hz}, \mathrm{H}-6), 6.35(1 \mathrm{H}, \mathrm{d}, J=2.4 \mathrm{~Hz}$, $\mathrm{H}-8), 6.33\left(1 \mathrm{H}, \mathrm{dd}, J=8.8,2.4 \mathrm{~Hz}, \mathrm{H}-5^{\prime}\right), 6.32(1 \mathrm{H}, \mathrm{d}$, $\left.J=2.8 \mathrm{~Hz}, \mathrm{H}-3^{\prime}\right), 5.62(1 \mathrm{H}, \mathrm{dd}, J=13.6,2.8 \mathrm{~Hz}, \mathrm{H}-2), 2.98$ $(1 \mathrm{H}, \mathrm{dd}, J=17.2,12.8 \mathrm{~Hz}, \mathrm{H}-3 \mathrm{a}), 2.68(1 \mathrm{H}, \mathrm{dd}, J=17.2,2.8$ $\mathrm{Hz}, \mathrm{H}-3 \mathrm{~b}) ;{ }^{13} \mathrm{C}-\mathrm{NMR}\left(100 \mathrm{MHz}, \mathrm{CD}_{3} \mathrm{OD}, \delta_{\mathrm{C}}\right) 194.32$ (C-4), 166.44 (C-8a), 166.09 (C-7), 159.69 (C-2'), 157.09 (C-4'),
129.89 (C-5), 128.81 (C-6'), 118.17 (C-1'), 114.94 (C-4a), 111.65 (C-6'), 107.76 (C-5'), 103.81 (C-8), 103.41 (C-3'), 76.49 (C-2), $44.08(\mathrm{C}-3)$.

화합물 3 (methyl $\beta$-resorcylate): Brown oil (in $\mathrm{CHCl}_{3}$ ); $\mathrm{E} /$ MS $m / z 168[\mathrm{M}]^{+}$; IR (KBr, v) 3380, 1666, $1621 \mathrm{~cm}^{-1} ;{ }^{1} \mathrm{H}-$ NMR $\left(400 \mathrm{MHz}, \mathrm{CDCl}_{3}, \delta_{\mathrm{H}}\right) 7.67(1 \mathrm{H}, \mathrm{d}, J=8.8 \mathrm{~Hz}, \mathrm{H}-6)$, $6.37(1 \mathrm{H}$, br.s, H-3), 6.35 (1H, br.d, $J=8.8 \mathrm{~Hz}, \mathrm{H}-5), 3.87$ $\left(3 \mathrm{H}, \quad \mathrm{s}, \mathrm{H}-\mathrm{OCH}_{3}\right) ;{ }^{13} \mathrm{C}-\mathrm{NMR}\left(100 \mathrm{MHz}, \mathrm{CDCl}_{3}, \delta_{\mathrm{C}}\right) 170.39$ (C-7), 163.55 (C-4), 162.67 (C-2), 131.76 (C-6), 108.02 (C5), $105.42(\mathrm{C}-1), 103.05(\mathrm{C}-3), 51.94\left(\mathrm{C}-\mathrm{OCH}_{3}\right)$.

화합물 4 ((Z)-oxyresveratrol-4- $O-\beta$-D-glucopyranoside): Brown amorphous powder (in $\mathrm{MeOH}) ;[\alpha]_{\mathrm{D}}^{25}-20.2^{\circ}(c \quad 0.39, \mathrm{MeOH})$; m.p. 200-205; EI/MS $m / z 406[\mathrm{M}]^{+}$; IR (KBr, v) 3391, 1608, $1594 \mathrm{~cm}^{-1}$; ${ }^{1} \mathrm{H}-\mathrm{NMR} \quad\left(400 \mathrm{MHz}, \mathrm{CD}_{3} \mathrm{OD}, \delta_{\mathrm{H}}\right) 7.02(1 \mathrm{H}, \mathrm{d}$, $J=8.8 \mathrm{~Hz}, \mathrm{H}-6), 6.55(1 \mathrm{H}, \mathrm{d}, J=2.4 \mathrm{~Hz}, \mathrm{H}-3), 6.52(1 \mathrm{H}, \mathrm{d}$, $J=12.0 \mathrm{~Hz}, \mathrm{H}-\alpha), 6.40(1 \mathrm{H}, \mathrm{dd}, J=8.8,2.4 \mathrm{~Hz}, \mathrm{H}-5), 6.34$ $(1 \mathrm{H}, \mathrm{d}, J=12.0 \mathrm{~Hz}, \mathrm{H}-), 6.21\left(2 \mathrm{H}, \mathrm{d}, J=2.4 \mathrm{~Hz}, \mathrm{H}-2^{\prime}, 6^{\prime}\right)$, $6.07\left(1 \mathrm{H}, \mathrm{t}, J=2.4 \mathrm{~Hz}, \mathrm{H}-4^{\prime}\right), 4.80(1 \mathrm{H}, \mathrm{d}, J=7.6 \mathrm{~Hz}, \mathrm{H}-1 ")$, $3.88(1 \mathrm{H}, \mathrm{dd}, J=12.4,2.4 \mathrm{~Hz}, \mathrm{H}-6 \mathrm{a}), 3.69(1 \mathrm{H}, \mathrm{dd}, J=$ 12.4, $5.2 \mathrm{~Hz}, \mathrm{H}-6 " \mathrm{~b}), 3.38\left(1 \mathrm{H}, \mathrm{m}, \mathrm{H}-3^{\prime \prime}\right), 3.36(1 \mathrm{H}, \mathrm{m}, \mathrm{H}-$ 5"), $3.36(1 \mathrm{H}, \mathrm{m}, \mathrm{H}-2 "), 3.34$ (1H, m, H-4"); ${ }^{13} \mathrm{C}-\mathrm{NMR}$ $\left(100 \mathrm{MHz}, \mathrm{CD}_{3} \mathrm{OD}, \delta_{\mathrm{C}}\right) 159.47$ (C-4), 159.23 (C-3'), 159.23 (C-5'), 157.16 (C-2), 140.67 (C-1'), 131.81 (C-6), 129.95 (C$\beta), 126.25(\mathrm{C}-\alpha), 119.96$ (C-1), 110.01 (C-5), 108.52 (C-2'), 108.52 (C-6'), 104.70 (C-3), 102.32 (C-4'), 102.15 (C-1"), 78.11 (C-3"), 78.02 (C-5"), 74.85 (C-2"), 71.37 (C-4"), 62.55 (C-6").

화합물 4 (Brown amorphous powder)는 EI/MS에서 $m / z 406$ $[\mathrm{M}]^{+}$의 분자이온 peak가 관측되어 분자량을 406 으로 결정하였 다. IR 스펙트럼으로부터 수산기 $\left(3391 \mathrm{~cm}^{-1}\right)$ 와 이중결합 $(1608$, $\left.1594 \mathrm{~cm}^{-1}\right)$ 의 작용기를 갖는 것으로 확인되었다. ${ }^{1} \mathrm{H}-\mathrm{NMR}(400$ $\mathrm{MHz}, \mathrm{CD}_{3} \mathrm{OD}$ ) spectrum에서는 저자장영역에서 olefin methine proton signal $\left(\delta_{\mathrm{H}} 6.40,1 \mathrm{H}, \mathrm{dd}, J=8.8,2.4 \mathrm{~Hz}, \mathrm{H}-5\right)$ 이 $\delta_{\mathrm{H}}$ 
$7.02(1 \mathrm{H}, \mathrm{d}, J=8.8 \mathrm{~Hz}, \mathrm{H}-6)$ signal과 vicinal coupling $\left(J_{3}\right)$ 함이 확인되었고, $\delta_{\mathrm{H}} 6.55(1 \mathrm{H}, \mathrm{d}, J=2.4 \mathrm{~Hz}, \mathrm{H}-3)$ 과는 long range $\left(J_{4}\right)$ coupling을 확인하였다. 이로 미루어 1,2,4-삼치환 benzene ring 1 개를 예측하였다. 또한, olefin methine proton signal $\left(\delta_{\mathrm{H}} 6.21,2 \mathrm{H}, \mathrm{d}, J=2.4 \mathrm{~Hz}, \mathrm{H}-2{ }^{\prime}, 6\right.$; $6.07,1 \mathrm{H}, \mathrm{t}$, $\left.J=2.4 \mathrm{~Hz}, \mathrm{H}-4^{\prime}\right)$ 3개가 관측되어 1,3,5-삼치환 benzene ring 1 개를 예측하였다. 또한, olefin methine proton signal $\left(\delta_{\mathrm{H}} 6.52\right.$, $1 \mathrm{H}, \mathrm{d}, J=12.0 \mathrm{~Hz}, \mathrm{H}-\mathrm{a} ; 6.34,1 \mathrm{H}, \mathrm{d}, J=12.0 \mathrm{~Hz}, \mathrm{H}-\mathrm{b}) 2$ 개 를 관측하였으며, 이 두 signal의 coupling constant $(J)$ 값이 $12.0 \mathrm{~Hz}$ 인 것으로부터 $c i s$ 배향의 이중결합을 확인할 수 있었 다. 산소가 치환된 영역에서는 한 개의 hemiacetal proton signal $\left(\delta_{\mathrm{H}} 4.80,1 \mathrm{H}, \mathrm{d}, J=7.6 \mathrm{~Hz}, \mathrm{H}-1 "\right)$ 이 관측되었으며 $J$ 값 이 $7.6 \mathrm{~Hz}$ 로부터 $\beta$-결합하고 있음을 확인하였고, 당에서 유래하 는 4 개의 oxygenated methine 및 1 개의 oxygenated methylene signal이 $\delta_{\mathrm{H}}$ 3.34-3.38에서 관측되었다. 따라서, proton 데이터를 종합하여, cis 배향의 stilbene 골격에 4개의 수산기, 한 개의 당 이 결합된 화합물로 예측하였다. ${ }^{13} \mathrm{C}-\mathrm{NMR}\left(100 \mathrm{MHz}, \mathrm{CD}_{3} \mathrm{OD}\right)$ spectrum에서 당을 포함하여 총 20개의 탄소 signal이 관측되었 으며, 저자장영역에서 stilbene 유래의 oxygenated olefin quaternary carbon signal $\left(\delta_{\mathrm{C}} 159.47, \mathrm{C}-4\right.$; 159.23, C-3'; 159.23, C-5'; $157.16, \mathrm{C}-2) 4$ 개와, olefin quaternary carbon signal $\left(\delta_{\mathrm{C}} 140.67\right.$, C-1'; 119.96, C-1) 2개, olefin methine carbon signal $\left(\delta_{\mathrm{C}}\right.$ 131.81, C-6; 129.95, C-b; 126.25, C-a; 110.01, C-5; 108.52, C-2'; 108.52, C-6'; 104.70, C-3; 102.32, C-4') 8개가 관측되었 다. 수산기영역에서는 당 유래의 hemiacetal carbon signal $\left(\delta_{\mathrm{C}}\right.$ $102.15, \mathrm{C}-1 "), 4$ 개의 oxygenated methine carbon signal $\left(\delta_{\mathrm{C}}\right.$ 78.11, C-3"; 78.02, C-5"; 74.85, C-2"; 71.37, C-4"), 1개의 oxygenated methylene carbon signal $\left(\delta_{\mathrm{C}} 62.55\right.$, C-6")의 chemical shift로부터 당의 구조가 $\beta$-glucopyranose임을 확인하 였다. 당의 결합위치를 확인 하기 위하여 heteronuclear multiple bond connectivity ( $\mathrm{HMBC}$ )를 측정하였으며, 그 결과 anomer proton signal $\left(\delta_{\mathrm{H}} 4.80, \mathrm{H}-1 "\right)$ 이 oxygenated olefin quaternary carbon signal $\left(\delta_{\mathrm{C}} 159.47, \mathrm{C}-4\right)$ 과 cross peak를 보여 glucose 가 4번에 결합하고 있음을 알 수 있었다. 이를 토대로 문헌 (Hakim 등, 2004)과 비교하여, 화합물 4를 (Z)-oxyresveratrol-4$O$ - $\beta$-D-glucopyranoside로 구조동정하였다(Fig. 1).

Norartocarpanone (1), 2',4',7-trihydroxy-(2S)-flavanone (2) 및 methyl $\beta$-resorcylate (3)은 이전에 상백피로부터 분리 보고된 바 있으나, (Z)-oxyresveratrol-4-O- $\beta$-D-glucopyranoside (4)는 상백 피에서는 이번에 처음으로 분리, 동정하였다. Norartocarpanone (1)과 2',4',7-trihydroxy-(2S)-flavanone (2)는 tyrosinase 억제활성 (Kang 등, 2011; Zheng 등, 2009)이 보고되어 있다.

\section{초 록}

뽕나무(Morus alba L.) 뿌리껍질을 실온에서 $80 \% \mathrm{MeOH}$ 수용 액으로 추출하고 이 추출물을 EtOAC, $n-\mathrm{BuOH}$, 그리고 $\mathrm{H}_{2} \mathrm{O}$ 분획으로 나누었다. EtOAc 및 $n-\mathrm{BuOH}$ 분획에 대하여 silica gel, ODS 및 Sephadex LH-20 column chromatography를 반 복 실시하여 4종의 phenolic 화합물을 분리, 정제하였다. NMR, $\mathrm{IR}$, 및 $\mathrm{EI} / \mathrm{MS}$ 등을 해석하여, norartocarpanone (1), 2',4',7trihydroxy-(2S)-flavanone (2), methyl- $\beta$-resorcylate (3), 그리고 (Z)-oxyresveratrol-4-O- $\beta$-D-glucopyranoside (4)로 각각 구조동정 하였다. 화합물 (Z)-oxyresveratrol-4-O- $\beta$-D-glucopyranoside (4) 는 상백피로부터는 이번 실험에서 처음으로 분리되었다.

Keywords 뽕나무 · 상백피 · nuclear magnetic resonance, phenolic compound · (Z)-oxyresveratrol-4-O- $\beta$-D-glucopyranoside

감사의 글 본 연구는 한국식품연구원(20140398U0054101S00100)에서 지 원한 연구비로 수행 되었음.

\section{References}

Hakim EH, Achmad SA, Aimi N, Indrayanto G, Kitajima M, and Makmur L (2004) Regioselective glucosylation of oxyresveratrol by cell suspension cultures of Solanum mammosum. J Chem Res 2004, 706-7.

Jung JW, Park JH, Jung YJ, Lee CH, Han D, and Baek NI (2014) Isolation and identification of triterpenoids from the Mulberry (Morus alba) root bark. J Appl Biol Chem 57, 295-9.

Kang KB, Kim SD, Kim TB, Jeong EJ, Kim YC, Sung JH et al. (2011) Tyrosinase inhibitory constituents of Morus bombycis cortex. Natl Prod Res 17, 198-201.

Zheng ZP, Chem S, Wang S, Wang XC, Cheng KW, Wu JJ et al. (2009) Chemical components and tyrosinase inhibitors from the twigs of Artocarpus heterophyllus. J Agric Food Chem 57, 6649-55. 